\title{
Efficient Classification of Birds and Drones Considering Real Observation Scenarios Using FMCW Radar
}

\author{
Se-Won Yoon ${ }^{1} \cdot$ Soo-Bum Kim² $\cdot$ Joo-Ho Jung ${ }^{3} \cdot$ Sang-Bin Cha ${ }^{1} \cdot$ Young-Seok Baek ${ }^{4} \cdot$ \\ Bon-Tae $\mathrm{Koo}^{4} \cdot \mathrm{In}-\mathrm{Oh} \mathrm{Choi}^{5} \cdot$ Sang-Hong Park ${ }^{1, *}$
}

\begin{abstract}
In this study, we consider real observation scenarios and propose an efficient method to accurately distinguish drones from birds using features obtained from their micro-Doppler (MD) signatures. In the simulations conducted using a rotating-blade model and a flappingwing model, the classification result degraded significantly due to the diversity of both drones and birds, but a combination of features obtained for longer observation times significantly improved the accuracy. MD bandwidth was found to be the most efficient feature, but sufficient observation time was required to exploit the period of time-varying MD as a useful feature.
\end{abstract}

Key Words: ATR, Feature Extraction, Micro-Doppler, Micro-Motion, UAV.

\section{INTRODUCTION}

Unmanned aerial vehicles (drones) have wide applications, such as military reconnaissance, areal mapping, and environmental monitoring. However, drones can also be used for illegal surveillance or military purposes. Therefore, efficient methods are required to detect drones and to distinguish them from similar targets, mainly birds.

Automatic target recognition (ATR) [1] using radar signals can effectively detect and classify drones. ATR recognizes a target by analyzing the data collected from radar, which uses wideband electromagnetic signals. ATR has been used to classify enemy jets, tanks, and other weapons in warfare. It analyzes a compressed wideband signal to extract two signatures: a highresolution range profile (HRRP) and an inverse synthetic aper- ture radar (ISAR) image, which represents the radar crosssection (RCS) distribution information [1-3]. However, these two methods might be inefficient when the size and RCS of the target are similar or if the target is engaged in additional motion during the coherent processing interval (CPI). Drones have similar RCS to that of birds (approximately $20 \mathrm{dBsm}$ ), which impedes the classification performed using RCS and HRRP. Furthermore, ISAR images can be significantly blurred due to the time-varying micro-Doppler (MD) effect [4] caused by the rotating blades of a drone or the flapping wings of a bird.

MD represents the time-varying micro-motion of a target [4-8]; thus, MD analysis has high potential for use in ATR. The basic principle of MD analysis is that the rapid mechanical rotation and vibration components of a rigid body cause additional Doppler frequency modulation on the returned radar

Manuscript received November 25, 2020 ; Revised January 12, 2021 ; Accepted March 2, 2021. (ID No. 20201125-191J)

${ }^{1}$ Pukyong National University, Busan, Korea.

${ }^{2}$ Radsys Co. Ltd., Daegu, Korea.

${ }^{3}$ Institute of Security Convergence Technology, Kookmin University, Seoul, Korea.

${ }^{4}$ Electronics and Telecommunications Research Institute, Daejeon, Korea.

${ }^{5}$ Department of Electronics and Communications Engineering, Korea Maritime \& Ocean University, Busan, Korea.

"Corresponding Author: Sang-Hong Park (e-mail: radar@pknu.ac.kr)

This is an Open-Access article distributed under the terms of the Creative Commons Attribution Non-Commercial License (http://creativecommons.org/licenses/by-nc/4.0) which permits unrestricted non-commercial use, distribution, and reproduction in any medium, provided the original work is properly cited.

(c) Copyright The Korean Institute of Electromagnetic Engineering and Science. 
signal. This additional modulation can significantly blur the ISAR image in the cross-range direction and change the amplitude of the range bin that corresponds to the blade position in the HRRP, thereby degrading the ATR accuracy [9-12]. However, $\mathrm{MD}$ can effectively distinguish drones from birds because they both have distinct micro-motions and therefore different MD signatures. A drone blade is engaged in two-dimensional (2D) rotation, so its $\mathrm{MD}$ in the time-frequency (TF) domain is symmetric, and the rotation period is well-represented $[5,6]$. In addition, the MD bandwidth is relatively large due to the large rotation speed of the blade, and blade flashes appear due to the blade RCS. In contrast, the MD of the bird is caused by wing flapping, which is not symmetric and has a narrower bandwidth than the signature of the drone blade. Therefore, $\mathrm{MD}$ can be used to achieve high ATR accuracy to distinguish drones from birds.

In this study, we evaluated how various MD features of a frequency-modulated continuous-wave (FMCW) radar signal [13] affect the accuracy of discrimination between birds and drones. For this purpose, we constructed a radar signal by using a rotating blade and a bird wing composed of a plate and an ellipsoid, each of which have an analytically defined RCS. In addition, efficient features for classifying drones and birds were proposed by expanding the features previously proposed in a conference [14]. Classifications were conducted for various observation scenarios and the usefulness of each feature was analyzed. In addition, the improvement achieved by the feature fusion was analyzed, and the accuracies of a nearest-neighbor classifier $\left(\mathrm{NNC}_{1}\right)$ [15] and a neural network classifier $\left(\mathrm{NNC}_{2}\right)$ [16] were compared. The classification results showed that the MD bandwidth was the most adequate for short observation time $T_{o b}$ but that to exploit the time-periodic nature of $\mathrm{MD}, T_{o b}$ must be extended. The correct classification ratio $\mathrm{Pc} \approx 100 \%$ was achieved by using only three features. $\mathrm{NNC}_{1}$ was found to be more appropriate than $\mathrm{NNC}_{2}$ for drone-bird classification.

\section{MATHEMATICAL ModELING AND PROPOSED METHOD}

\section{Mathematical Modeling of the Rotating Drone Blade and Flapping Bird Wing}

A drone blade can be modeled using two collinear plates of width $2 a_{b}$ and length $2 b_{b}$, which are rotated around the $\mathrm{x}$-axis, one by $\pm 45^{\circ}$ and another by $-45^{\circ}$ (Fig. 1). The coordinates of a blade tip rotating clockwise at frequency $f_{b}$ around the $\mathrm{z}$-axis are obtained as

$$
\begin{aligned}
\left(\begin{array}{l}
x_{b} \\
y_{b} \\
z_{b}
\end{array}\right) & =R(t)\left[\begin{array}{c}
2 a \\
0 \\
0
\end{array}\right]=\left[\begin{array}{ccc}
\cos \left(2 \pi f_{b} t\right) & \sin \left(2 \pi f_{b} t\right) & 0 \\
-\sin \left(2 \pi f_{b} t\right) & \cos \left(2 \pi f_{b} t\right) & 0 \\
0 & 0 & 1
\end{array}\right]\left[\begin{array}{c}
2 a \\
0 \\
0
\end{array}\right] \\
& =\left(\begin{array}{c}
2 a \cos \left(2 \pi f_{b} t\right) \\
-2 a \sin \left(2 \pi f_{b} t\right) \\
0
\end{array}\right)
\end{aligned}
$$

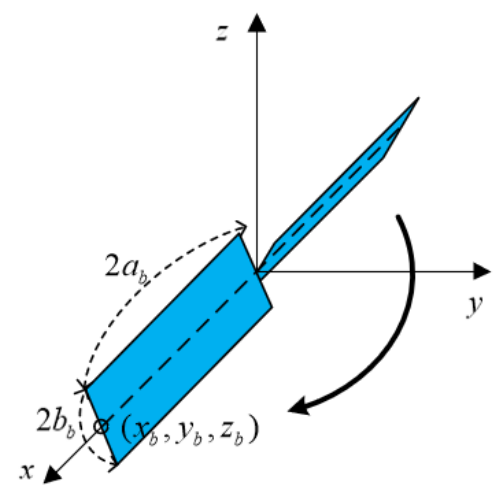

Fig. 1. Drone blade model.

Assuming that a plate is observed at a line-of-sight (LOS) vector composed of an azimuth angle $\phi$ and an elevation $\theta$ (Fig. 2), the RCS of the flat plate rotated by $45^{\circ}$ is analytically given as [13]:

$$
\mathrm{RCS}=\left(\cos \theta \frac{\sin x_{k}}{x_{k}} \frac{\sin y_{k}}{y_{k}}\right),
$$

where

$$
\begin{gathered}
x_{k}=(2 \pi / \lambda) a_{b} \sin \theta \sin \phi, y_{k}=(2 \pi / \lambda) b_{b}, \\
\theta=\cos ^{-1}\left(-L O S \bullet V_{3}\right), \phi=\tan ^{-1}\left(\frac{\left|L O S \bullet V_{4}\right|}{\left|L O S \bullet V_{1}\right|}\right)
\end{gathered}
$$

with

$$
\begin{gathered}
V_{1}=\left[\begin{array}{l}
1 \\
0 \\
0
\end{array}\right], V_{2}=\left[\begin{array}{ccc}
\cos \left(90^{\circ}\right) & 0 & -\sin \left(90^{\circ}\right) \\
0 & 1 & 0 \\
\sin \left(90^{\circ}\right) & 0 & \cos \left(90^{\circ}\right)
\end{array}\right] \bullet V_{1}=\left[\begin{array}{l}
0 \\
0 \\
1
\end{array}\right], \\
V_{3}=\left[\begin{array}{ccc}
1 & 0 & 0 \\
0 & \cos \left(45^{\circ}\right) & \sin \left(45^{\circ}\right) \\
0 & -\sin \left(45^{\circ}\right) & \cos \left(45^{\circ}\right)
\end{array}\right] \bullet V_{2}=\left[\begin{array}{c}
1 \\
1 / \sqrt{2} \\
1 / \sqrt{2}
\end{array}\right], \\
V_{4}=\left[\begin{array}{ccc}
1 & 0 & 0 \\
0 & \cos \left(-45^{\circ}\right) & \sin \left(-45^{\circ}\right) \\
0 & -\sin \left(-45^{\circ}\right) & \cos \left(-45^{\circ}\right)
\end{array}\right] \bullet V_{2}=\left[\begin{array}{c}
1 \\
-1 / \sqrt{2} \\
1 / \sqrt{2}
\end{array}\right]
\end{gathered}
$$

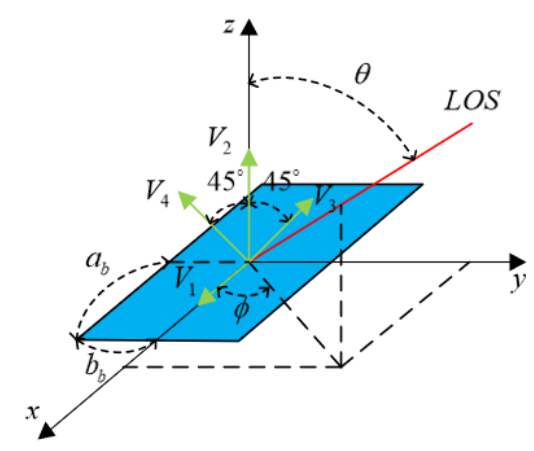

Fig. 2. Observation geometry to calculate the RCS of a blade. 
Using the same $R(t)$ as that in (1), $V_{i}$ for $i=1,2,3,4$ change accordingly as

$$
V=R(t) \bullet V_{i} \text { for } \mathrm{i}=1,2,3,4,
$$

so the RCS of the rotating blade can be obtained at each LOS by using (2).

The bird motion was modeled using the upper and back muscles of the wing (Fig. 3). With the same sinusoidal frequency $f_{w}$, the analysis assumed that the lower muscle tip at $\left(x_{1}, y_{1}, z_{1}\right)$ rotated by $\theta_{1}=40^{\circ} \cos \left(2 \pi f_{w} t\right)+15^{\circ}$ and the upper muscle tip at $\left(x_{2}, y_{2}, z_{2}\right)$ rotated by $\theta_{2}=30^{\circ} \cos \left(2 \pi f_{w} t\right)+40^{\circ}$. The forward-backward movement of the upper wing was modeled by rotating $\left(x_{2}, y_{2}, z_{2}\right)$ by $\phi_{w}=20^{\circ} \sin \left(2 \pi f_{w} t\right)+40^{\circ}$ in the $x-y$ plane. Thus, the two-muscle position can be expressed as

$$
\left(\begin{array}{l}
x_{1} \\
y_{1} \\
z_{1}
\end{array}\right)=\left(\begin{array}{l}
0 \\
r_{1} \cos \theta_{1} \\
y_{1} \tan \theta_{1}
\end{array}\right),\left(\begin{array}{l}
x_{2} \\
y_{2} \\
z_{2}
\end{array}\right)=\left(\begin{array}{l}
r_{2} \tan \phi_{w} \\
y_{1}+r_{2} \cos \phi_{w} \cos \left(\theta_{1}-\theta_{2}\right) \\
z_{1}+\left(y_{2}-y_{1}\right) \tan \left(\theta_{1}-\theta_{2}\right)
\end{array}\right),
$$

where $r_{1}$ and $r_{2}$ are

$$
\begin{aligned}
& r_{1}=\sqrt{x_{1}^{2}+y_{1}^{2}+z_{1}^{2}} \\
& r_{2}=\sqrt{\left(x_{2}-x_{1}\right)^{2}+\left(y_{2}-y_{1}\right)^{2}+\left(z_{2}-z_{1}\right)^{2}} .
\end{aligned}
$$

The wing muscle can have various shapes but our main concern is the MD analysis; thus, the muscle was modeled as an ellipsoid (Fig. 4). As in the case of a flat plate, the RCS of the ellipsoid observed at an observation angle $\theta_{w}$ with respect to the major axis is analytically given as

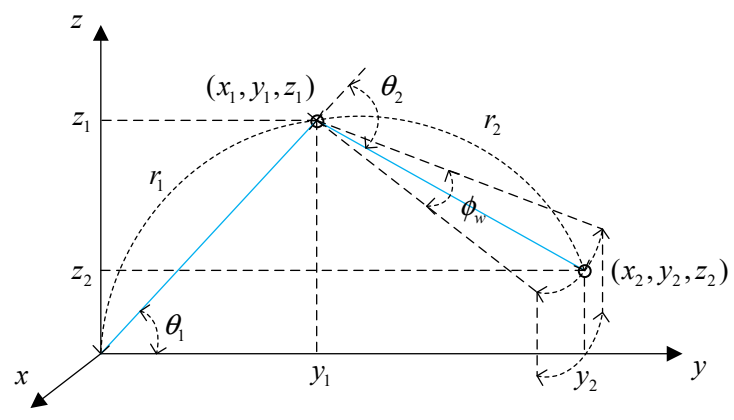

Fig. 3. Geometry of bird-wing micro-motion.

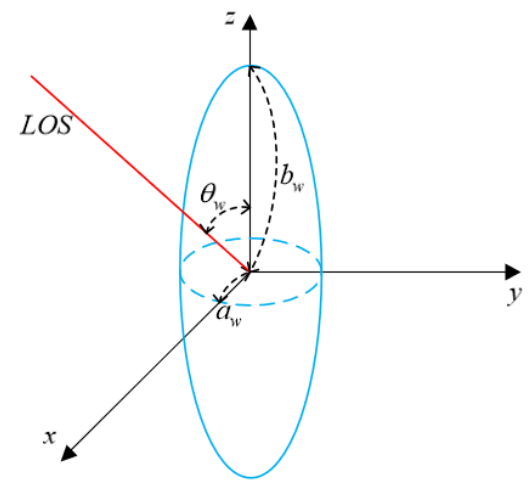

Fig. 4. Ellipsoid used for bird muscle and observation geometry.

$$
\mathrm{RCS}=\frac{\pi\left(a_{w} / 2\right)^{4}\left(b_{w} / 2\right)^{2}}{\left(\left(a_{w} / 2 \times \sin \theta_{w}\right)^{2}+\left(b_{w} / 2 \times \cos \theta_{w}\right)^{2}\right)^{2}},
$$

where $a_{w}$ and $b_{w}$ are the lengths of the minor and major axes, respectively [13]. Assuming that the vector of the major axis of the lower and upper muscles is $\bar{p}_{i}(i=1$ for the lower and 2 for the upper muscle), $\theta_{w}$ is calculated as

$$
\theta_{i}=\cos ^{-1}\left(\frac{\bar{p}_{i} \cdot \operatorname{LOS}}{\left|\bar{p}_{i}\right||L O S|}\right) \text { for } i=1 \text { and } 2
$$

where

$$
\bar{p}_{1}=\left[\begin{array}{l}
0 \\
y_{1} \\
z_{1}
\end{array}\right], \bar{p}_{2}=\left[\begin{array}{c}
x_{2} \\
y_{2}-y_{1} \\
z_{2}-z_{1}
\end{array}\right]
$$

are the centers of muscles 1 and 2 , respectively.

\section{Radar Signal Modeling and Principle of $M D$}

In this study, we used an FMCW signal to form the received radar signal. FMCW is a widely used, low-cost and low-power radar system that can be implemented in a small system and is widely used for short-range detection and imaging. The FMCW radar continuously transmits and receives a chirp signal within a given period $T_{\text {chirp }}$ [13] (Fig. 5).

The transmitted chirp signal, which is repeated at intervals $T_{\text {chirp }}$, is expressed as

$$
s_{T}(t)=\exp \left(j 2 \pi\left(f_{0} t+\frac{K_{r} t^{2}}{2}\right)\right)
$$

where $f_{0}$ is the minimum frequency and $K_{r}$ is the chirp rate (Fig. 5). The bandwidth $B, K_{r}$, and $T_{\text {chirp }}$ are related as

$$
K_{r}=\frac{B}{T_{\text {chirp }}} \text {. }
$$

The received chirp from a scatterer at $r$ is given by

$$
s_{R}(t)=\exp \left(j 2 \pi\left(f_{0}(t-\tau)+\frac{K_{r}(t-\tau)^{2}}{2}\right)\right),
$$

where the time delay $\tau=2 r / c$ and $c$ is the speed of the light. For the bistatic observation scenario, $2 r$ is replaced by $r_{t}+r_{r}$, where $r_{t}$ is the distance from the transmitting radar to the target and $r_{r}$ is the distance to the target receiving radar.

Dechirping the received chirp yields an intermediate signal:

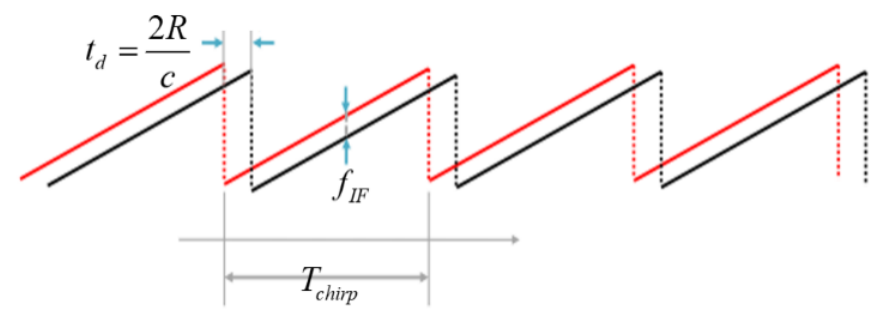

Fig. 5. Transmitted and received FMCW signals. 


$$
s_{I F}(t)=\exp \left(j 2 \pi\left(f_{c} \tau+K_{r} \tau t-\frac{K_{r} \tau^{2}}{2}\right)\right),
$$

where $K_{r} \tau$ is the beat frequency:

$$
f_{b}=K_{r} \tau=\frac{2 K_{r}}{c} r
$$

where the residual video phase $K_{r} \tau^{2} / 2$ has been removed.

Assuming that a target with an RCS $A_{0}$ is observed for a CPI $T_{C P I},(11)-(15)$ are used for each slow time $t_{s}=k T_{\text {chip }}$ during $T_{C P I}$. Thus, the received signal can be expressed as

$$
\begin{aligned}
& s_{0}\left(t, t_{s}\right) \\
& =A_{0} \exp \left\{-j \frac{4 \pi f_{0} R\left(t_{s}\right)}{c}\right\} \exp \left(j \pi K_{r}\left(t-\frac{2 R\left(t_{s}\right)}{c}\right)^{2}\right),
\end{aligned}
$$

where $R\left(t_{s}\right)$ is the distance to the target at $t_{s .}$. Dechirping changes $s_{0}\left(t, t_{s}\right)$ to $s_{0 d}\left(t, t_{s}\right)$ as

$$
\begin{aligned}
s_{0 d}\left(t, t_{s}\right)= & A_{0} \exp \left\{-j \frac{4 \pi f_{0} R\left(t_{s}\right)}{c}\right\} \\
& \times \exp \left(j 2 \pi\left(f_{c} \tau+K_{r} \tau t-\frac{1}{2} K_{r} \tau^{2}\right)\right) .
\end{aligned}
$$

If the third term in the above equation is ignored [17], a Fourier transform (FT) from the $t$-domain to the $f$-domain yields a dechirped signal in HRRP at $t_{s}$ (i.e., $s_{r}\left(f, t_{s}\right)$ :

$$
\begin{aligned}
& s_{r}\left(f, t_{s}\right) \\
& =A_{0} \exp \left\{-j \frac{4 \pi\left(f_{0}+f\right)}{c} R\left(t_{s}\right)\right\} p_{r}\left(f-K_{r} \tau\right),
\end{aligned}
$$

where $p_{r}(\cdot)$ is a sinc function formed by a rectangular window of length $T_{\text {chipp. }}$. The down-range location of the target can be calculated by determining the peak of $p_{r}(\cdot)$.

The signal $s_{M D}\left(t_{s}\right)=s_{r}\left(K_{r} \tau_{p}, t_{s}\right)$, where $\tau_{p}$ is the time delay of the signal reflected from the target. $s_{M D}\left(t_{s}\right)$ is the time-varying signal of the target caused by the micro-motion and sampled at $t_{s}$. However, $s_{M D}\left(t_{s}\right)$ is time-varying and thus should be analyzed in the TF domain rather than the frequency domain. In this study, we used the short-time Fourier transform (STFT), which is simple to implement and free from cross-term interference. STFT transforms $s_{M D}\left(t_{s}\right)$ to $\operatorname{MD}[18,19]$ :

$$
M D\left(t_{s}, f_{s}\right)=\int_{-\infty}^{\infty} s_{M D}(\zeta) W^{*}\left(\zeta-t_{s}\right) e^{-j 2 \pi f_{s} \zeta} d \zeta .
$$

where $f_{s}$ is the frequency and $W$ is the window function. To reduce the sidelobe caused by the rectangular window, we used the Hamming window. In discrete form, (22) is expressed as

$$
M D[p, q]=\sum_{k=0}^{L-1} s[k] W[k-p] e^{-(2 \pi q k) / L},
$$

where $k$ is the time index, $L$ is the window length, $p$ is the sampled time index, and $q$ is the sampled frequency index.
The MD image was transformed to the TF domain (Fig. 6). The received signal (19) was dechirped to obtain HRRP for each $t_{s}$. Then, the range bin with the highest amplitude was selected and STFT was applied to obtain the MD image in the TF domain.

\section{Feature Vectors for Classification}

The following features are proposed in this paper (Table 1).

The frequency $f_{r s}$ of the MD signal variation in the time domain represents the rotation frequency of the drone blades; thus, $f_{r s}$ is used as a feature and is defined as

$$
f_{r c s}=\arg \max _{f}(\mid \operatorname{FT}(r c s(\tau) \mid)),
$$

where $\operatorname{rcs}(\tau)=\left|s_{M D}(\tau)\right|$.

The frequency $f_{b}$ of the MD change in the time domain dif-

\begin{tabular}{|c|c|c|c|}
\hline Feature & Definition & Feature & Definition \\
\hline$f_{1}$ & $f_{r c s}$ (monostatic) & $f_{2}$ & $f_{b}$ (monostatic) \\
\hline$f_{3}$ & $f_{v}($ monostatic $)$ & $f_{4}$ & $f_{r s s}$ (bistatic) \\
\hline$f_{5}$ & $f_{b}$ (bistatic) & $f_{6}$ & $f_{v}$ (bistatic) \\
\hline$f_{7}$ & ent (monostatic) & $f_{8}$ & ent $t_{v}$ (monostatic) \\
\hline$f_{9}$ & $e n t_{2 D}$ (monostatic) & $f_{10}$ & enth (bistatic) \\
\hline$f_{11}$ & entv (bistatic) & $f_{12}$ & $e n t_{2 D}$ (bistatic) \\
\hline$f_{13}$ & $f_{p k, h}($ monostatic $)$ & $f_{14}$ & $f_{p k, v}($ monostatic) \\
\hline$f_{15}$ & $f_{p k, 2 D}$ (monostatic) & $f_{16}$ & $f_{p k, h}$ (bistatic) \\
\hline$f_{17}$ & $f_{p k, v}($ bistatic $)$ & $f_{18}$ & $f_{p k, 2 D}$ (bistatic) \\
\hline$f_{19}$ & $\operatorname{cor}_{ \pm}($monostatic $)$ & $f_{20}$ & cor $_{ \pm}$(bistatic) \\
\hline$f_{21}$ & $\operatorname{cor}_{m b}$ & - & - \\
\hline
\end{tabular}
fers considerably between the drone and the bird; thus, this change is used as a feature. To obtain $f_{b}$, the frequency-domain image $I_{f f}$ is obtained by the FT of the MD image in (23) in the time domain as

$$
I_{f f}=\operatorname{FT}_{q}(M D[p, q]),
$$

where $\mathrm{FT}_{q}$ is the FT in $q$ for each $p$. Then, $f_{b}$ is obtained as

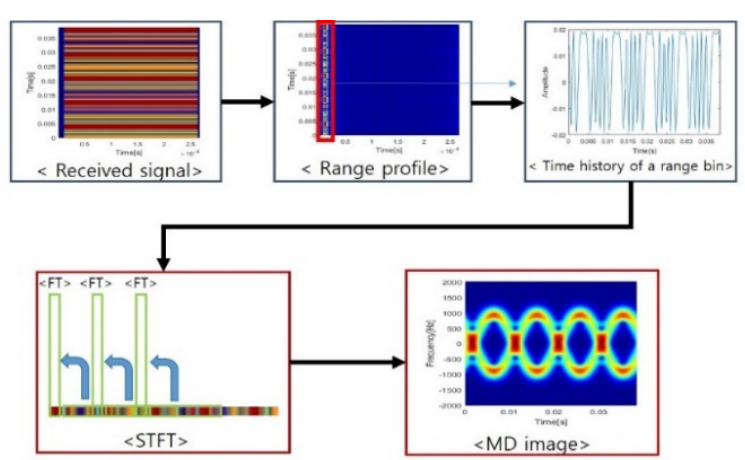

Fig. 6. Procedure to obtain the MD image.

Table 1. Features proposed in this paper 


$$
f_{h}=\max \left(\left|\sum_{p} I_{f f}[p, q]\right|\right) .
$$

Due to the difference in the rotation speed, the MD bandwidth $f_{v}$ is used as a feature and can be defined as

$$
f_{v}=\operatorname{fmax}\left(\left|\sum_{q} I_{f f}[p, q]\right|, \gamma\right),
$$

where $f_{\max }(s(k), \gamma)$ is the maximum $k$ corresponding to a value larger than $\gamma \%$ of the maximum $s(k)$.

The MD frequencies in the positive and negative directions differ between drones and birds. Drones have symmetric rotation, whereas birds do not; thus, the $2 \mathrm{D}$ correlation cor \pm of $\pm \mathrm{MD}$ frequencies is used as a feature. The correlation cor $r_{ \pm}$is defined as

$$
c o r_{ \pm}=\sum_{m=0}^{M-1} \sum_{n=0}^{N-1}\left(\frac{X[m, n]-\bar{X}}{\sqrt{V(X)}}\right)\left(\frac{Y[-m,-n]-\bar{Y}}{\sqrt{V(Y)}}\right),
$$

where $X$ and $Y$ are the MD images clipped from $I_{f f}$ for positive and negative MD frequencies, respectively; $\bar{X}$ and $\bar{Y}$ are the averages of $X$ and $Y$; and $V(\cdot)$ is the variance.

Drone blades rotate symmetrically in the $x-y$ plane, so the monostatic/bistatic MD images of MD drones are similar, whereas bird wings flap in the $z-y$ plane. The correlation cor $_{m b}$ obtained using the monostatic and bistatic images is used as a feature:

$$
\begin{aligned}
& \operatorname{cor}_{m b} \\
& =\sum_{m=0}^{M-1} \sum_{n=0}^{N-1}\left(\frac{I_{f f, m}[m, n]-\bar{I}_{f f, m}}{\sqrt{V\left(I_{f f, m}\right)}}\right)\left(\frac{I_{f f, b}[-m,-n]-\bar{I}_{f f, b}}{\sqrt{V\left(I_{f f, b}\right)}}\right),
\end{aligned}
$$

where $I_{f f, m}$ and $I_{f f, b}$ are monostatic and bistatic $I_{f f}$, respectively, and $\bar{I}_{f f, m}$ and $\bar{I}_{f f, b}$ are the averages of $I_{f f, m}$ and $I_{f f, b}$.

The MD of the drone is widespread; thus, the peak value of the normalized MD frequency is small. However, the MD of the bird is narrowly spread; thus, the peak is large. Therefore, the peak value $f_{p k, v}$ is used as a feature:

$$
f_{p k, v}=\max \left(\frac{Z_{v}}{\sum_{n} Z_{v}[n]}\right),
$$

where $Z_{v}[n]=\sum_{q}\left|I_{f f}[p, q]\right|$ is the projected sum onto the vertical axis.

$f_{p k, b}$ is used as a feature and is defined as

$$
f_{p k, h}=\max \left(\frac{Z_{h}}{\sum_{n} Z_{h}[n]}\right),
$$

where $Z_{b}[n]=\sum_{q}\left|I_{f f}[p, q]\right|$, which is the projected sum onto the horizontal axis.

Similarly, the peak value $f_{p k, 2 D}$ in the normalized $I_{f f}$ is

$$
f_{p k, 2 D}=\max \left(I_{N}\right),
$$

where

$$
I_{N}=\frac{\left|I_{f f}\right|}{\sum_{p} \sum_{q}\left|I_{f f}[p, q]\right|} .
$$

Entropy is a good measure of disorder [20]. It increases with the width of a profile but not with a narrowly distributed profile. Therefore, the entropy ent $t_{v}$ of $Z_{v}$ is used as a feature and can be expressed as

$$
\text { ent } t_{v}=\sum_{n}\left(-Z_{v}[n] \times \log \left(Z_{v}[n]\right)\right) .
$$

The entropy of $Z_{b}$ is also used as a feature:

$$
\text { ent }_{h}=\sum_{n}\left(-Z_{h}[n] \times \log \left(Z_{h}[n]\right)\right) .
$$

Similarly, ent ${ }_{2 D}$, as the $2 \mathrm{D}$ entropy of $I_{N}$, is used as a feature:

$$
\text { ent }_{2 D}=\sum_{p} \sum_{q}\left(-I_{N}[p, q] \times \log \left(I_{N}[p, q]\right)\right) .
$$

For the convenience of combining features, they are defined as $f_{i}$ for $i=1,2, \ldots, 21$ (Table 1 ).

\section{Construction of the Training Database, Classifier, and Overall Procedure}

To obtain a classification ratio of approximately $100 \%$, the training database should be constructed for all combinations of azimuth and elevation angles. In addition, the direction of flight and frequencies of blade rotation and wing flapping should be considered; thus, the computation time and memory space are huge. To circumvent these problems, we constructed a training database for flight scenarios [2] (Fig. 7). We uniformly sampled the three-dimensional space (training space) and assumed that

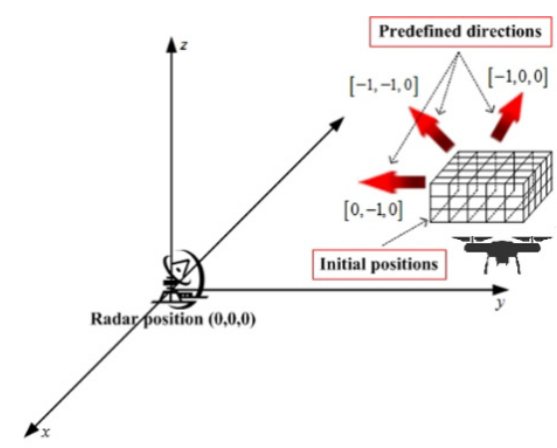

Fig. 7. Method to construct the training database. 
the target flew at a given velocity in a given direction, starting from each given grid point. Then, the MD image was obtained and stored in the training database.

To consider the variation in flight direction and MD frequency, the MD image was obtained at each grid point by uniformly sampling the flight direction within $\pm \theta_{t}$ in increments of $\Delta \theta_{t}$. In addition, for each flight direction, $f_{b}$ of the drone blade was sampled between $f_{b, 1}$ and $f_{b, 2}$ in increments of $\Delta f_{b}$, and $f_{w}$ of the bird wing was sampled between $f_{w, 1}$ and $f_{w, 2}$ in increments of $\Delta f_{w}$. In the bistatic observation scenario, a receiving radar was located at a distance $r_{b}$ from the transmitting radar, and the training database was constructed using the same grid points and motion parameters.

The classifiers used in this study were $\mathrm{NNC}_{1}$ and $\mathrm{NNC}_{2}$, and their classification accuracies were compared. $\mathrm{NNC}_{1}$ uses a simple Euclidean norm as [15]

$$
\hat{i}=\min _{i} g_{i}\left(\bar{x}_{u}\right) \text {, }
$$

with

$$
g_{i}\left(\bar{x}_{u}\right)=\left\|\bar{x}_{u}-\bar{f}_{i}\right\|,
$$

where $\bar{x}_{u}$ is a test vector and $\bar{f}_{i}$ is a training vector that belongs to the $i$ th class. The class $i$ that yields the minimum $\mathrm{Eu}^{-}$ clidean norm is the class that includes $\overline{x_{u}}$.

As $\mathrm{NNC}_{2}$, we constructed a neural network composed of two hidden layers: the first layer had five nodes and the second had three [16] (Fig. 8). Training was conducted using a backpropagation algorithm; the output was set to "1" for drones and "0" for birds. Because the decrease in the training error after 5,000 iterations was slight, 10,000 iterations were conducted to prevent time consumption. The classification was performed by following the general target recognition procedure (Fig. 9). When the test

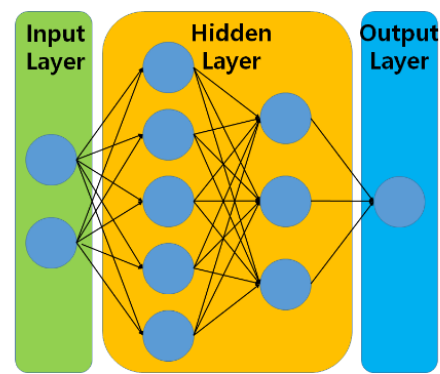

Fig. 8. Architecture of $\mathrm{NNC}_{2}$ used in this paper.

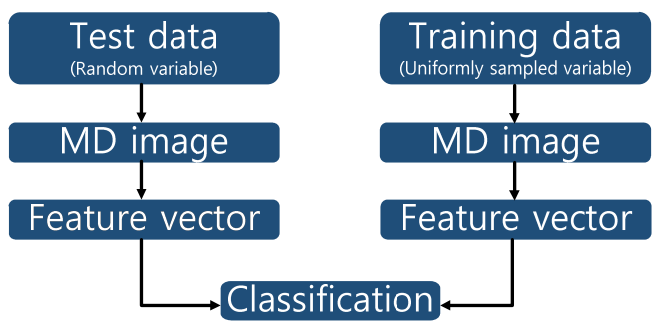

Fig. 9. Classification procedure. data were obtained, the target location and motion parameter were not known; thus, the target was randomly positioned in the training space, and the micro-motion was modeled using a parameter that was randomly set within the range of that $\mathrm{pa}^{-}$ rameter in the training data. Then, an MD image was obtained following Fig. 6. Classification was conducted using the defined features (Table 1), and the importance of each feature and the classification accuracy of the two classifiers were evaluated.

\section{SIMULATION RESULT}

\section{Simulation Condition}

Simulations were conducted using an X-band FMCW radar with center frequency $=9.6 \mathrm{GHz}$ and pulse-repetition frequency $(\mathrm{PRF})=4 \mathrm{kHz}$, which are the specifications of a tracking radar that we are developing. To analyze the importance of the features that represent the periodicity of the MD signal, observations were conducted for two values of TCPI. The first was 0.0385 seconds, which is the standard dwell time of the scanning radar, and the second was 1.001 seconds, so that a long observation time could exploit the periodicity of the MD signal. The bandwidth was assumed as $B=150 \mathrm{MHz}$; however, we reduced it to $0.150 \mathrm{MHz}$ for fast computation, because the MD image is not relevant to range resolution but sampling the target using PRF, which exists in a range bin (Fig. 10).

We assumed a normal-sized drone composed of four wings, each of which had four blades rotating at a frequency between $f_{b, 1}=40 \mathrm{~Hz}$ and $f_{b, 2}=50 \mathrm{~Hz}$. The blade dimensions were selected as $a_{b}=8 \mathrm{~cm}$ and $b_{b}=1.5 \mathrm{~cm}$ (Fig. 2). For the bird, we assumed a wing span $=60 \mathrm{~cm}$ (i.e., $a_{w}=b_{w}=15 \mathrm{~cm}$ ) at a flapping frequency $f_{w, 1}=4 \mathrm{~Hz}$ or $f_{w, 2}=7 \mathrm{~Hz}$. As mentioned above, the $\mathrm{MD}$ of a bird is very different from that of a drone (Fig. 11).

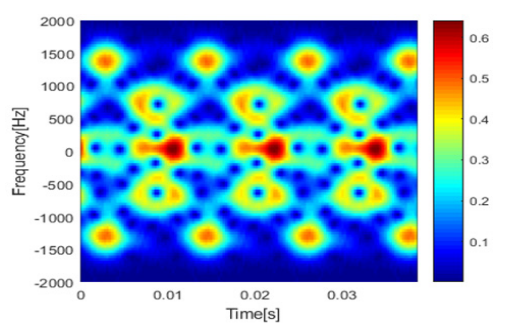

(a)

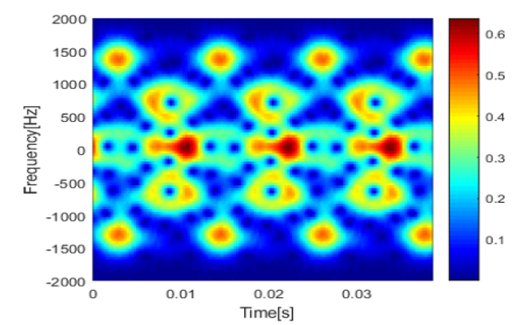

(b)

Fig. 10. Comparison of MD images obtained using bandwidths: (a) $150 \mathrm{MHz}$ and (b) $0.150 \mathrm{MHz}$. 


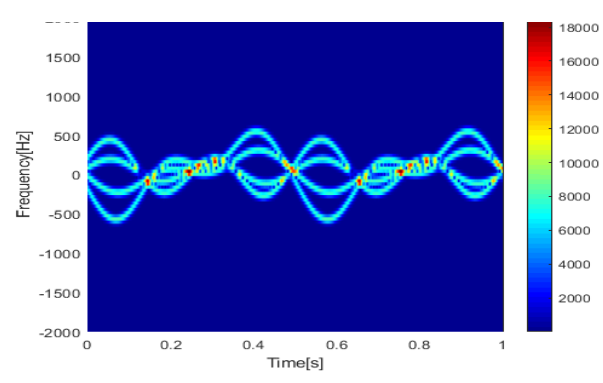

Fig. 11. MD image of the bird model.

Due to the asymmetric motion of a bird, its MD is not symmetric and blade flashes are not observed. In addition, the period of $\mathrm{MD}$ is much smaller than that of the blade.

In constructing the training database, the training space was divided into 250 subspaces by using a grid. The range on the $x$ axis between 300 and $1,500 \mathrm{~m}$ was divided into ten equal intervals; the range on the $z$-axis between 10 and $1,000 \mathrm{~m}$ was divided into five equal intervals; and the azimuth angle between $-45^{\circ}$ and $+45^{\circ}$ was divided into five equal intervals (Fig. 12(a)). At each grid point, the flight direction was set between $-\theta_{t}=-30^{\circ}$ and $+\theta_{t}=30^{\circ}$ in increments of $\Delta \theta_{t}=12^{\circ}$. For each flight direction on each grid point, the MD image was obtained by varying $f_{b}$ and $f_{w}$ in increments of $1 \mathrm{~Hz}$. The test data were randomly obtained. Identical simulations were conducted for bistatic observations with a receiving radar located at $1,000 \mathrm{~m}$ on the $y$ axis from the transmitting radar at the origin.

At a random position in the training space (i.e., $300 \mathrm{~m} \leq x \leq$ $1,500 \mathrm{~m}, 10 \mathrm{~m} \leq z \leq 100 \mathrm{~m}, 0^{\circ} \leq$ azimuth angle $\leq 45^{\circ}$ ), the target was flown in a random direction at $-30 \leq \theta_{t} \leq 30^{\circ}$ with a random micro-motion frequency (Fig. 12(b)). For $T_{C P I}=0.0385$ seconds, 2,500 test images were used per target; for $T_{C P I}=1.001$

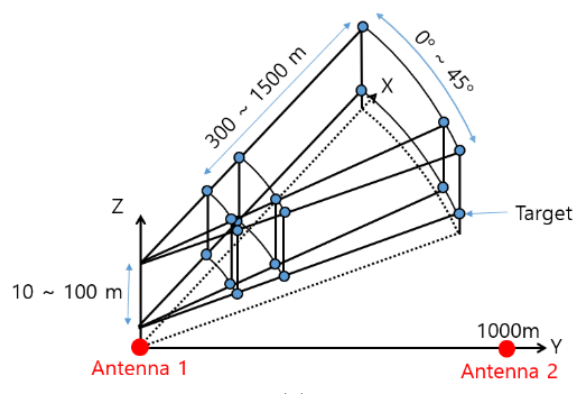

(a)

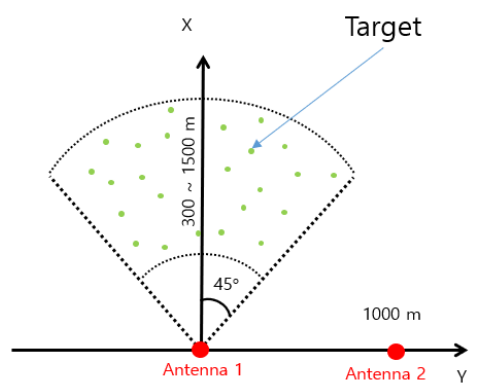

(b)

Fig. 12. (a) Training space and (b) test space. seconds, this number was decreased to 300 due to the increased computation time. As in the training phase, the bistatic test data were obtained using the same parameters. The classification accuracy was expressed as the correct classification percentage:

$$
P_{c}=M_{d} / M_{t e} \times 100 \%,
$$

where $M_{c}$ is the number of correct classifications and $M_{t e}$ is the number of test samples.

\section{Classification Results \\ 1) Classification results for the stationary target}

Classifications were performed assuming that the targets were stationary with the random micro-motion parameters at a randomly selected position. The signal-to-noise ratio (SNR) was varied from -15 to $20 \mathrm{~dB}$ in increments of $5 \mathrm{~dB}$, and $\mathrm{NNC}_{1}$ was used as the classifier. The $P_{c}$ values for both $T_{C P I}$ were proportional to the SNR, except for $f_{21}$ for $T_{C P I}=0.0385$ seconds. $P_{c}$ was approximately $100 \%$ at $\mathrm{SNR}=20 \mathrm{~dB}$ (Fig. 13). Comparing the features for each $T_{C P I}$, the highest $P_{s}$ at low SNRs were obtained using $f_{2}$ and $f_{5}$ (i.e., MD frequency $f_{b}$ for monostatic and bistatic scenarios), which is a rather unexpected result. These results are attributed to the fact that for the short $\mathrm{CPI}$, the frequency of MD change of the bird is approximately $0 \mathrm{~Hz}$ due to the slow variation in bird flapping, whereas the blade rotation is fast enough to yield a certain value of $f_{b}$. As a result, $P_{c}$ was high.

Comparing the results obtained at the two $T_{C P I}$ values, the features that exploited the MD periodicity and the projected sum onto the horizontal axis (i.e., $f_{1}, f_{7}, f_{10}, f_{13}, f_{16}$ ) were significantly improved at $T_{C P I}=1.0$ second because the increased observation time allowed the periodicity to be represented by FT along the time domain (= horizontal axis). $f_{2}$ and $f_{5}$ are also the features representing MD periodicity; however, the $P_{s}$ of these vectors for $T_{C P I}=1.0$ second were lower than those for $T_{C P I}=$ 0.0385 seconds because of the large zero-nonzero difference.

Likewise, $f_{21}$ increased for $T_{C P I}$ because the increased observation time represented the MD image in detail. The entropyrelated features $\left(f_{7}-f_{12}\right)$ yielded a high $P_{c}$ value but were sensitive to noise.

\section{2) Classification results for the moving target}

To represent a real observation scenario, the first set of classifications was performed to study the effect of velocity $v$ and acceleration $a$ of a moving target by randomly selecting $0 \leq v$ $\leq 10 \mathrm{~m} / \mathrm{s}$ and $0 \leq a \leq 10 \mathrm{~m} / \mathrm{s}^{2}$.

As $v$ of the rigid body shifted the MD and $a$ tilted the MD upward or downward, the test MD image was significantly different from the training image (Fig. 14). Other simulation conditions were the same as those mentioned in Section III-2-1.

The classification results demonstrated that $v$ and $a$ should be accurately estimated and compensated for (Fig. 15). Compared 


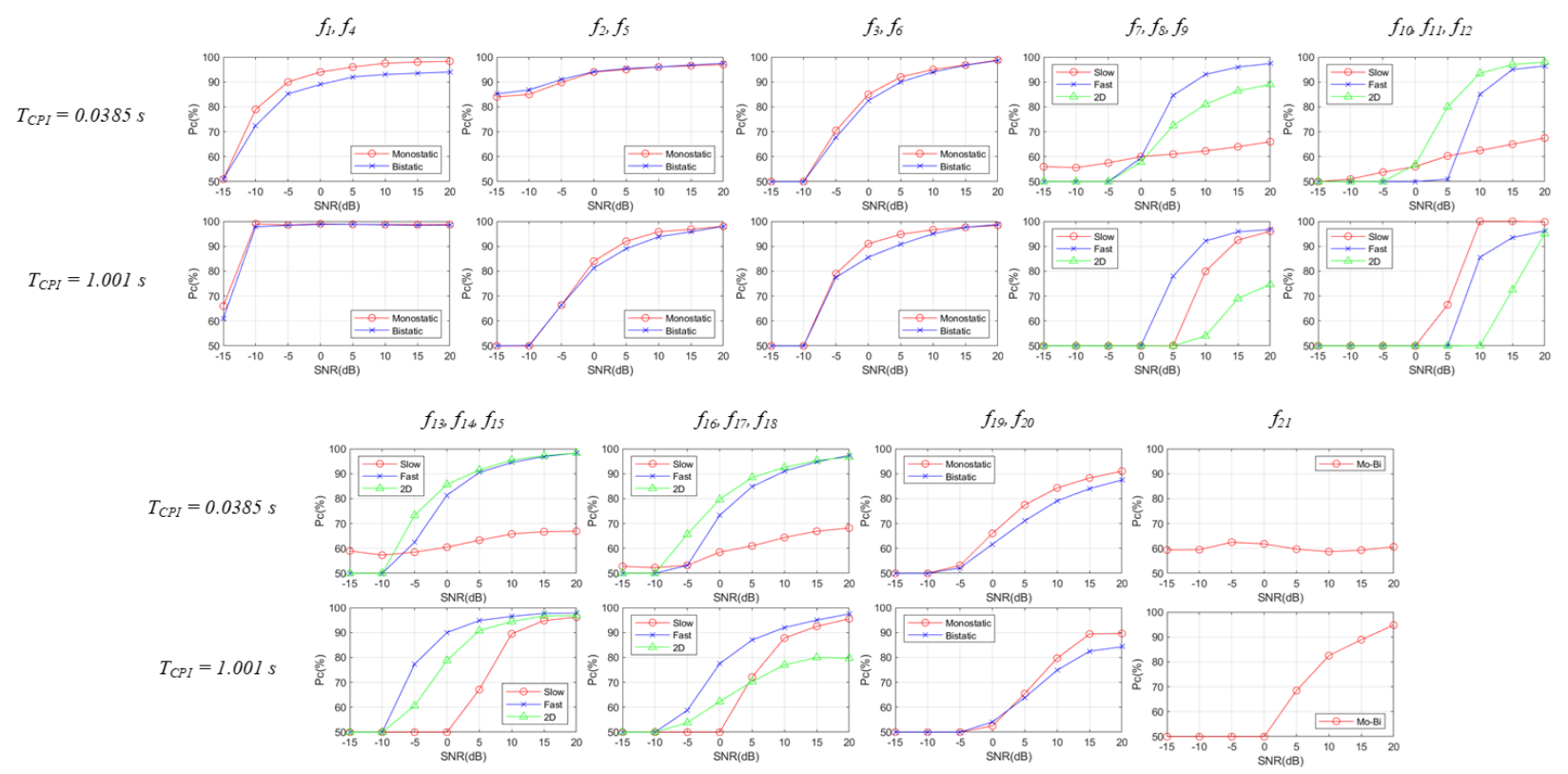

Fig. 13. $P_{c}$ for the stationary target (see Table 1 for the meaning of each feature).

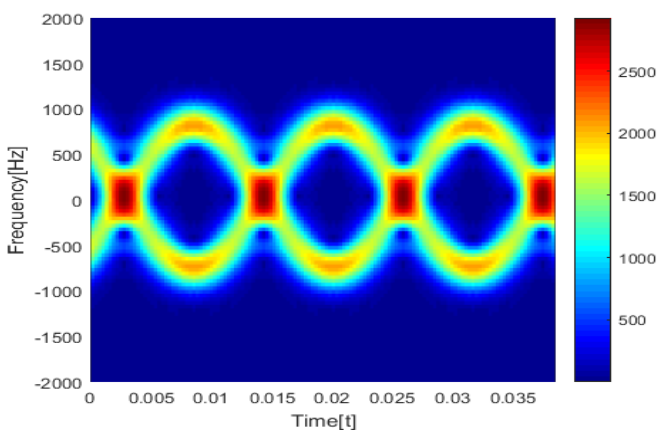

(a)

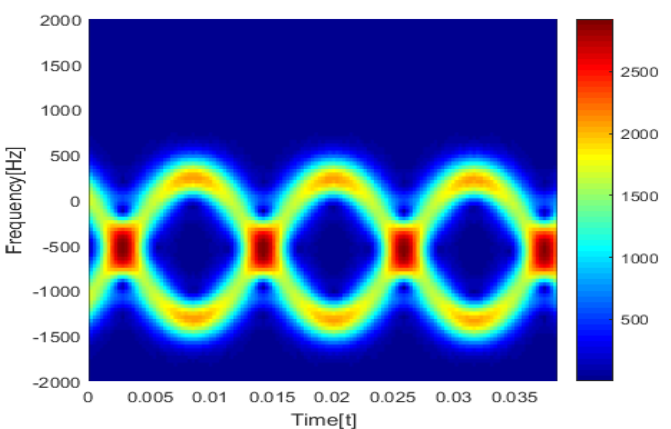

(b)

Fig. 14. Comparison of MD images of the stationary target (a) and moving target (b).

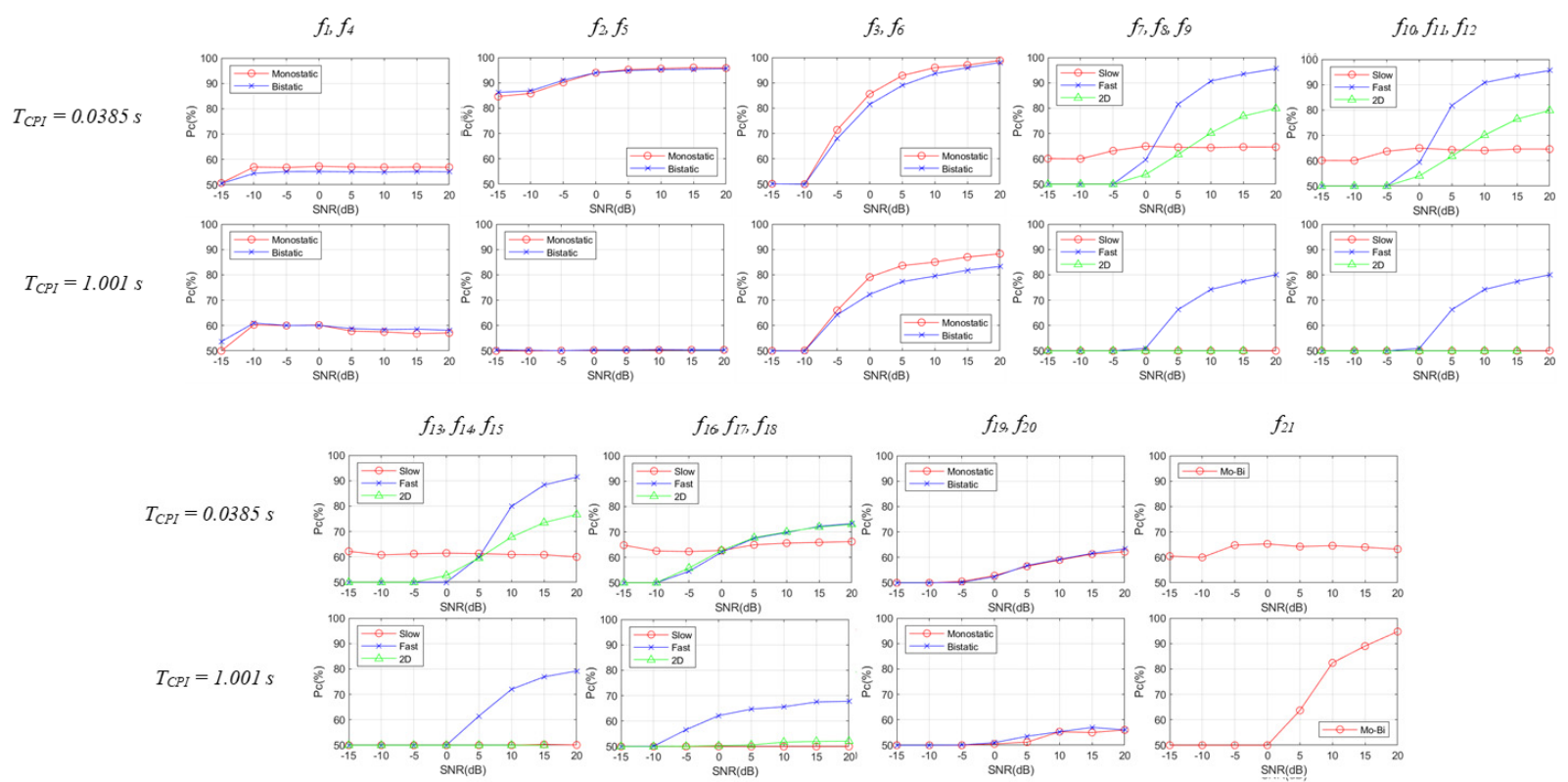

Fig. 15. $P_{c}$ s for the moving target (see Table 1 for the meaning of each feature). 
to the result of the stationary target (Fig. 13), $P_{c}$ of the features decreased significantly. Due to the change in MD frequency caused by $v$ and $a$, the RCS frequency varied; thus, $f_{1}$ and $f_{4}$ yielded poor $P_{c}$. In addition, $P_{c}$ of the features $\left(f_{7}, f_{10}, f_{13}, f_{16}\right)$ using the horizontally projected sum decreased considerably due to the tilted MD image in the TF domain; the amount of decrease was larger at $T_{C P I}=1.001$ seconds than that at $T_{C P I}=$ 0.0385 seconds because the tilt of the MD frequency in the TF domain was larger at $T_{C P I}=1.001$ seconds than that at $T_{C P I}=$ 0.0385 seconds. Thus, the test $I_{f f}$ became totally different from the training $I_{f f} s$ after FT on the horizontal axis.

$T_{C P I}$ affected several $P_{c}$ values. At a short $T_{C P I}$, the $P_{c}$ values were high because these features are nonzero for a bird and close to zero for a drone. However, at a long $T_{C P I}$, the frequency of the MD change affected by $v$ and $a$ was represented by FT, so $P_{c}$ decreased to approximately $50 \%$. The MD bandwidth $\left(f_{3}\right.$ and $f_{6}$ ) was less affected because the MD bandwidth of the drone was much larger than that of the drone. The entropy obtained using the projected sum onto the vertical axis $\left(f_{7}\right.$ and $f_{10}$, "fast") was less affected by $v$ and $a$ than the MD bandwidth because the entropy is not determined by the MD bandwidth but by the overall distribution. The $P_{c}$ values of $f_{19}$ and $f_{20}$ were also affected by $v$ and $a$, and those of $f_{21}$ increased for $T_{C P I}$ due to the similarity between the monostatic and bistatic MD images for the increased observation time.

The second set of classifications was performed to study the effect of multiple targets because birds and drones may fly in groups with similar motion parameters. The variables $v$ and $a$ were assumed to have been perfectly compensated for, the number of targets was randomly selected between 1 and 5 inclusive, and the classifications were conducted using the same parame- ters as those used in the first classification.

Because the effects of $v$ and $a$ were completely removed, $P_{c}$ (Fig. 16) was slightly lower than the values shown in Fig. 13. The $P_{c}$ values for both $T_{C P I}$ values were proportional to SNR except for $f_{21}$ for $T_{C P I}=0.0385$ seconds. $f_{2}$ and $f_{5}$, which represent MD frequency $f_{b}$, yielded the highest $P_{c}$ at low SNRs due to zero and nonzero MD frequencies. $f_{1}, f_{7}, f_{10}, f_{13}$, and $f_{16}$, which represent the projected sum onto the horizontal axis, improved significantly for $T_{C P I}=1.0$ second due to the increased observation time. $f_{2}$ and $f_{5}$ for $T_{C P I}=1.0$ second yielded lower $P_{c}$ than those for $T_{C P I}=0.0385$ seconds because of the large zerononzero difference. The entropy-related features $\left(f_{T}-f_{12}\right)$ yielded high $P_{c}$ but were sensitive to noise, and $f_{21}$ increased for $T_{C P I}$ due to the increased observation time.

\section{3) Classification results for the fusion of features}

To demonstrate the improvement in feature fusion, classifications were conducted by fusing the features. Combinations of 21 features were used as the feature vector, and $P_{s}$ of $\mathrm{NNC}_{1}$ and $\mathrm{NNC}_{2}$ were compared for various $\mathrm{SNRs}$ using the training and test data same as those presented in Section III-2-1. The number of combinations was large, many of which had $P_{c} \approx$ $100 \%$, so combinations of two and three among the 21 features were analyzed, and the optimal combinations that yielded $P_{c} \approx$ $100 \%$ were found.

For combinations of two features, and considering the sum of $P_{c}$ at $\mathrm{SNR}=0 \mathrm{~dB}$ and $20 \mathrm{~dB}$, the best five combinations obtained $P_{c} \geq 95 \%$ for $\mathrm{SNR}=0 \mathrm{~dB}$ and $P_{c} \geq 98 \%$ for $\mathrm{SNR}=20$ $\mathrm{dB}$ (Fig. 17). As in the classifications conducted above, $P_{c}$ was proportional to SNR. For $T_{C P I}=0.0385$ seconds, $\mathrm{NNC}_{1}$ using $f_{2}+f_{5}, f_{2}+f_{15}, f_{2}+f_{18}, f_{5}+f_{15}$, and $f_{5}+f_{18}$ yielded high $P_{c} \approx 100 \%$

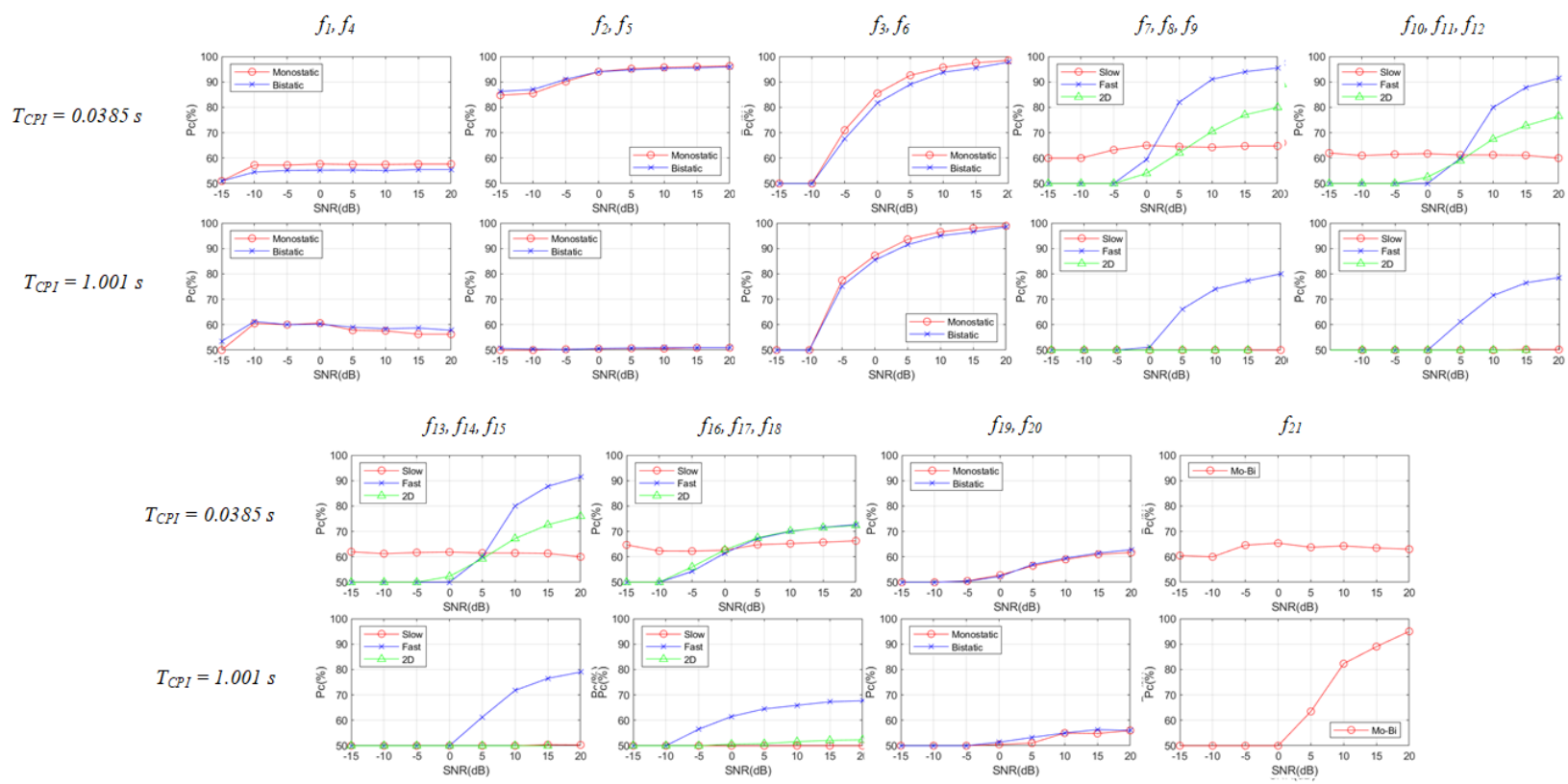

Fig. 16. $P_{s}$ for the multiple target (see Table 1 for the meaning of each feature). 

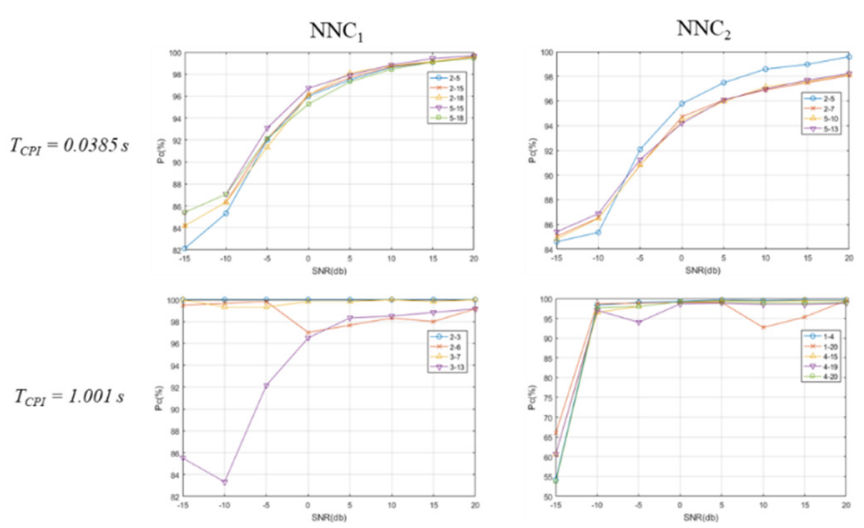

Fig. 17. Fusion result for two combinations.
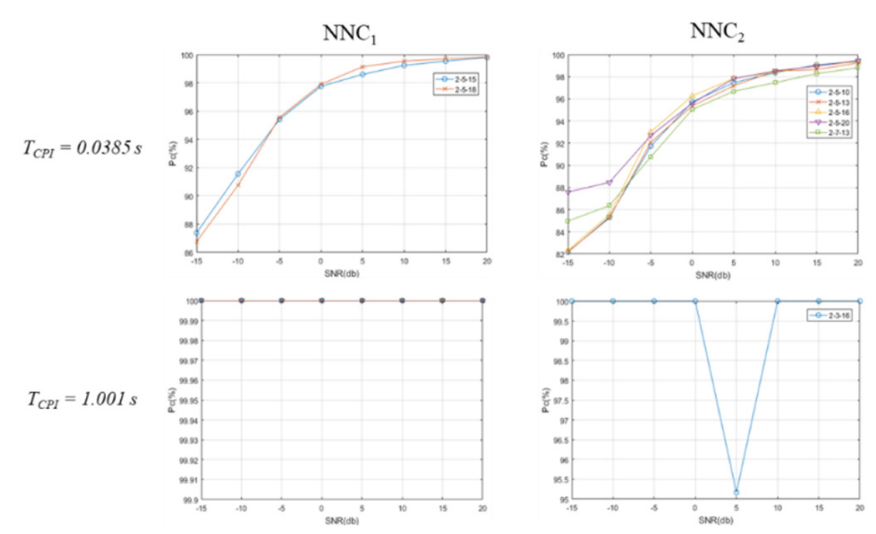

Fig. 18. Fusion result for three combinations.

for $\mathrm{SNR}=20 \mathrm{~dB}$, and for $\mathrm{NNC}_{2}, f_{2}+f_{5}, f_{2}+f_{7}, f_{5}+f_{10}$, and $f_{5}$ $+f_{13}$ were more effective than other features. Comparing $\mathrm{NNC}_{1}$ and $\mathrm{NNC}_{2}$, the $P_{c}$ of $\mathrm{NNC}_{1}$ was higher than that of $\mathrm{NNC}_{2}$. This is because the designed $\mathrm{NNC}_{2}$ (Fig. 8) was rather simple and the training data were over-fitted to $\mathrm{NNC}_{2}$; thus, $P_{c}$ was lower because of the lack of the generalization capability.

$P_{s}$ for $T_{C P I}=1.001$ seconds were much higher for both classifiers than for $T_{C P I}=0.0385$ seconds. For $\mathrm{NNC}_{1}, P_{s}$ of $f_{2}+f_{3}$, $f_{2}+f_{6}$, and $f_{3}+f_{7}$ were approximately $100 \%$ for all SNRs, and $f_{3}$ $+f_{13}$ was sensitive to noise. For $\mathrm{NNC}_{2}, P_{s}$ of $f_{1}+f_{4}, f_{1}+f_{20}, f_{4}+$ $f_{15}, f_{4}+f_{19}$, and $f_{4}+f_{20}$ were slightly lower than those of $\mathrm{NNC}_{2}$ at $\mathrm{SNR} \geq-10 \mathrm{~dB}$ but close to $100 \%$. The reason for the improvement was that for $T_{C P I}=1.001$ seconds, the features $f_{2}$ and $f_{7}$ for $\mathrm{NNC}_{1}$ and the features $f_{1}, f_{4}, f_{19}$, and $f_{20}$ better represented the periodicity of MD than that at $T_{C P I}=0.0385$ seconds.

Combinations of three features increased the $P_{c}$ value compared to those using combinations of two features, so the requirement to sift the classification results was changed. For $T_{C P I}$ $=0.0385$ seconds, the best five combinations that satisfied $P_{c} \geq$ $90 \%$ for $\mathrm{SNR}=-10 \mathrm{~dB}$ and $P_{c} \geq 98 \%$ for $\mathrm{SNR}=20 \mathrm{~dB}$ were displayed (Fig. 18). For $T_{C P I}=1.001$ seconds, the combinations for $\mathrm{NNC}_{1}$ that yielded $P_{c}=100 \%$ for all SNRs were used, and for $\mathrm{NNC}_{2}$, the combinations that yielded $P_{c} \geq 95 \%$ for all SNRs were used.
At $T_{C P I}=0.0385$ seconds $P_{c}$ improved only slightly at low SNRs because of the poor representation of the target by the features that exploit periodicity. Using $\mathrm{NNC}_{1}, f_{2}+f_{5}+f_{15}$ and $f_{2}$ $+f_{5}+f_{15}$ satisfied the requirement; using $\mathrm{NNC}_{2}, f_{2}+f_{5}+f_{10}, f_{2}$ $+f_{5}+f_{13}, f_{2}+f_{5}+f_{16}, f_{2}+f_{5}+f_{20}$, and $f_{2}+f_{7}+f_{13}$ satisfied the requirement. $P_{s}$ of $\mathrm{NNC}_{1}$ were slightly higher than those of $\mathrm{NNC}_{2}$, although the number of feature combinations for $\mathrm{NNC}_{1}$ was smaller than that for $\mathrm{NNC}_{2}$. For both classifiers, features $f_{2}$ and $f_{5}$ worked well because of the zero-nonzero MD relationship (Section III-2-1).

For $T_{C P I}=1.001$ seconds, 23 feature combinations for $\mathrm{NNC}_{1}$ yielded $P_{s}=100 \%$ for all SNRs: $f_{2}+f_{3}+f_{7}, f_{2}+f_{3}+f_{9}, f_{2}+f_{3}+$ $f_{10}, f_{2}+f_{3}+f_{12}, f_{2}+f_{3}+f_{13}, f_{2}+f_{3}+f_{14}, f_{2}+f_{3}+f_{15}, f_{2}+f_{3}+f_{16}$, $f_{2}+f_{3}+f_{18}, f_{2}+f_{3}+f_{19}, f_{2}+f_{3}+f_{20}, f_{2}+f_{3}+f_{21}, f_{2}+f_{7}+f_{9}, f_{2}$ $+f_{7}+f_{10}, f_{2}+f_{7}+f_{12}, f_{2}+f_{7}+f_{13}, f_{2}+f_{7}+f_{16}, f_{2}+f_{7}+f_{21}, f_{2}+f_{9}$ $+f_{13}, f_{2}+f_{10}+f_{13}, f_{2}+f_{13}+f_{16}, f_{2}+f_{13}+f_{21}, f_{5}+f_{6}+f_{7}, f_{5}+f_{6}+$ $f_{10}, f_{5}+f_{6}+f_{12}, f_{5}+f_{6}+f_{13}, f_{5}+f_{6}+f_{16}, f_{6}+f_{10}+f_{12}, f_{6}+f_{10}+f_{16}$, and $f_{6}+f_{10}+f_{21}$. The increased observation time made the features related to the horizontal component work very well, and the increased dimension provided additional information to separate the two classes further. For $\mathrm{NNC}_{2}, f_{2}+f_{3}+f_{16}$ satisfied the requirement; at $\mathrm{SNR}=5 \mathrm{~dB}, P_{c}$ was only $95.2 \%$ due to the lack of generalization capability, so further study is required to achieve high $P_{c}$.

\section{CONCLUSION}

This paper proposes efficient features to classify drones and birds by using an FMCW radar. We conducted simulations to evaluate the effectiveness of each feature. The radar signal was constructed using the analytically known RCS of the drone blade and bird wing. A training database that considered flight scenarios was constructed to reduce the required memory space and computation time. The simulation results suggested that features that represent the vertical component of the MD image can be used regardless of the observation time, but those that represent the horizontal component of the MD image are only effective if the observation is extended. For a target that had $v$ and $a, P_{c}$ decreased considerably, so the effects of $v$ and $a$ should be removed before the features are extracted. The proposed features were also robust to the existence of multiple targets, yielding a small decrement in $P_{c}$. High $P_{c}$ values were obtained by combining the features; as the observation time increased, the discriminant ability of the features related to the horizontal axis increased, so the improvement in $P_{c}$ increased. $\mathrm{NNC}_{1}$ yielded a higher $P_{c}$ than $\mathrm{NNC}_{2}$, which is attributable to the simple structure of the neural network. Further studies should be conducted to increase the number of neurons or use convolutional neural network structures.

The classification results were obtained assuming the specifica- 
tions of a radar system that we are currently developing. Therefore, noise, clutter, and antenna beam shape might make the modeled radar signal differ from the measured signal. The blade and wing models used here are only one of the wide range of models. The materials that constitute the blade might affect its RCS. Birds have wings of many shapes and flap them at a range of frequencies. Currently, we are conducting experiments to measure the $\mathrm{MD}$ signal of real flying drones and birds and to remove clutter from the measured signal. Further research on classification performed using the measured signal will be conducted, and used to improve the features and the classification methods.

This work was supported by the Institute of Information \& communications Technology Planning \& Evaluation (IITP) grant funded by the Korea government (MSIT) (No. 2018-0-00197, Development of ultra-low power intelligent edge SoC technology based on lightweight RISC-V processor). This research was supported by Basic Science Research Program through the National Research Foundation of Korea (NRF) funded by the Ministry of Science, ICT \& Future Planning (No. 2018R1D1A1B07044981). This research was supported by the National Research Foundation (NRF), Korea, under project BK21 FOUR (Smart Robot Convergence and Application Education Research Center).

\section{REFERENCES}

[1] P. Tait, Introduction to Radar Target Recognition. London, UK: The Institution of Engineering and Technology, 2005.

[2] S. H. Park, M. G. Joo, and K. T. Kim, "Construction of ISAR training database for automatic target recognition," Journal of Electromagnetic Waves and Applications, vol. 25, no. 11-12, pp. 1493-1503, 2011.

[3] S. K. Han, H. T. Kim, S. H. Park, and K. T. Kim, "Efficient radar target recognition using a combination of range profile and time-frequency analysis," Progress in Electromagnetics Research, vol. 108, pp. 131-140, 2010.

[4] V. C. Chen, F. Li, S. S. Ho, and H. Wechsler, "MicroDoppler effect in radar: phenomenon, model, and simulation study," IEEE Transactions on Aerospace and Electronic Systems, vol. 42, no. 1, pp. 2-21, 2006.

[5] T. Thayaparan, S. Abrol, E. Riseborough, L. J. Stankovic, D. Lamothe, and G. Duff, "Analysis of radar micro-Doppler signatures from experimental helicopter and human data," IET Radar, Sonar \& Navigation, vol. 1, no. 4, pp. 289$299,2007$.

[6] J. H. Jung, U. Lee, S. H. Kim, and S. H. Park, "MicroDoppler analysis of Korean offshore wind turbine on the L-band radar," Progress in Electromagnetics Research, vol.
143, pp. 87-104, 2013

[7] J. H. Jung, K. T. Kim, S. H. Kim, and S. H. Park, "MicroDoppler extraction and analysis of the ballistic missile using RDA based on the real flight scenario," Progress in Electromagnetics Research M, vol. 37, pp. 83-93, 2014.

[8] L. Liu, D. McLernon, M. Ghogho, W. Hu, and J. Huang, "Ballistic missile detection via micro-Doppler frequency estimation from radar return," Digital Signal Processing, vol. 22, no. 1, pp. 87-95, 2012.

[9] J. Li and H. Ling, "Application of adaptive chirplet representation for ISAR feature extraction from targets with rotating parts," IEE Proceedings-Radar, Sonar and Navigation, vol. 150, no. 4, pp. 284-291, 2003.

[10] L. Stankovic, I. Djurovic, and T. Thayaparan, "Separation of target rigid body and micro-Doppler effects in ISAR imaging," IEEE Transactions on Aerospace and Electronic Systems, vol. 42, no. 4, pp. 1496-1506, 2006.

[11] Q. Zhang, T. S. Yeo, H. S. Tan, and Y. Luo, "Imaging of a moving target with rotating parts based on the Hough transform," IEEE Transactions on Geoscience and Remote Sensing, vol. 46, no. 1, pp. 291-299, 2008.

[12] A. Ghaleb, L. Vignaud, and J. M. Nicolas, "MicroDoppler analysis of wheels and pedestrians in ISAR imaging," IET Signal Processing, vol. 2, no. 3, pp. 301-311, 2008.

[13] B. R. Mahafza, Radar Systems Analysis and Design Using MATLAB. Boca Raton, FL: CRC Press, 2000.

[14] S. Park, J. Jung, S. Cha, S. Kim, S. Youn, I. Eo, and B. Koo, "In-depth analysis of the micro-Doppler features to discriminate drones and birds," in Proceedings of 2020 International Conference on Electronics, Information, and Communication (ICEIC), Barcelona, Spain, 2020, pp. 1-3.

[15] R. O. Duda, P. E. Hart, and D. G. Stork, Pattern Classification, 2nd ed. New York, NY: John Wiley \& Sons Inc., 2001.

[16] C. C. Aggarwal, Neural Networks and Deep Learning: A Textbook. New York, NY: Springer, 2018.

[17] A. Meta, P. Hoogeboom, and L. P. Ligthart, "Signal processing for FMCW SAR," IEEE Transactions on Geoscience and Remote Sensing, vol. 45, no. 11, pp. 3519-3532, 2007.

[18] S. Qian and D. Chen, Joint Time-Frequency Analysis: Methods and Applications. Upper Saddle River, NJ: Prentice-Hall, 1996.

[19] S. Qian, Introduction to Time-Frequency and Wavelet Transforms. Upper Saddle River, NJ: Prentice-Hall, 2002.

[20] X. Li, G. Liu, and J. Ni, "Autofocusing of ISAR images based on entropy minimization," IEEE Transactions on Aerospace and Electronic Systems, vol. 35, no. 4, pp. 12401252, 1999. 


\section{Se-Won Yoon}

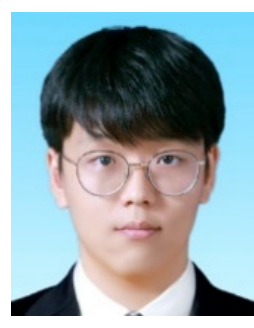

RCS prediction.

\section{Soo-Bum Kim}

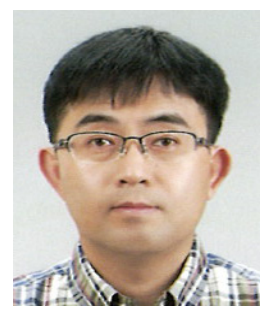

received his B.S. and M.S. degrees in electronic engineering from Pukyong National University, Busan, Korea, in 2017 and 2019, respectively, where he is currently working toward the Ph.D. degree in electronic engineering. His research interests are in the areas of radar target imaging and recognition, radar signal processing, target motion compensation, pattern recognition using artificial intelligence, and Co. Ltd., Daegu, Korea. His research interests include the system design and signal processing of radar and SAR systems.

\section{Joo-Ho Jung}

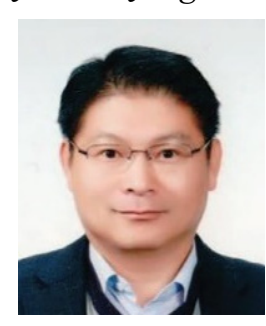

received his B.S. degree in the Korea Air Force Academy, Cheongju, Korea, in 1991 and his M.S. and $\mathrm{Ph} . \mathrm{D}$. degrees in electronic engineering from $\mathrm{Po}_{-}$ hang University of Science and Technology (POSTECH) in 1998 and 2007, respectively. From 2008 to 2012, he was a lieutenant colonel in the Defense Acquisition Program Administration, Seoul, Korea. In 2012, he joined the Department of Electrical Engineering, POSTECH, as a research associate professor, and in 2015, he was with Unmanned Technology Research Center, Korea Advanced Institute of Science and Technology, Daejeon. In 2020, he joined Kookmin University, Seoul, as the Director of EM Technology Research Center. His research interests are radar target recognition, radar signal processing, and electromagnetic analysis in wind farms by various military radars.
Sang-Bin Cha

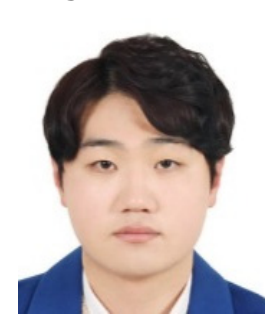

received his B.S. and M.S. degrees in electronic engineering from Pukyong National University, Busan, Korea, in 2017 and 2019, respectively, where he is currently working toward the Ph.D. degree in electronic engineering. His research interests are in the areas of radar target imaging and recognition, radar signal processing, target motion compensation, pattern recognition using artificial intelligence, RCS prediction, and electromagnetic analysis of the windfarm.
Young-Seok Baek

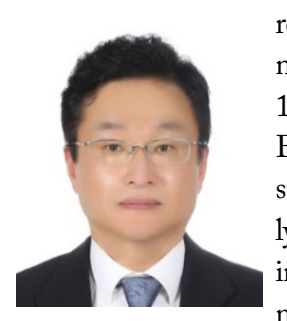

received his B.S., M.S. degrees in electronic engineering from Hanyang University, Seoul, Korea, in 1985 and 1987, respectively. In 1989, he joined the Electronics and Telecommunications Research Institute (ETRI), Daejeon, Korea, where he is currently a senior engineer. His research interests are $\mathrm{CAD}$ in semiconductor, digital design and verification methodology, wireless communication, face recogni-

tion, and radar signal processing.
Bon-Tae Koo

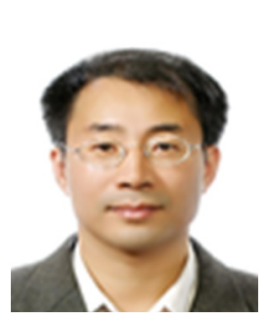

received his M.S. degrees in electrical engineering from Korea University, Seoul, Korea, in 1991. In 1991, he was with the System Semiconductor Division, Hyundai Electronics Company, Ichon, Korea, where he was involved in the chip design of video codec and DVB modem. From 1993 to 1995, he was with HEA, San Jose, USA, where he was responsible for the design of MPEG2 video codec chips. From 1996 to 1997, he was with TVCOM, San Diego, USA. In 1998, he joined Dongbu Electronics as a team leader with the system semiconductor Lab and focused on the methodology of semiconductor chip design. In 1999, he joined the Application SoC team, ETRI, where he is currently a team leader and his research activities included the chip design of MPEG4 video, T-DMB receiver, LTE femtocell modem, and DSP processor. His research activities focused on millimeter-wave AI radars.

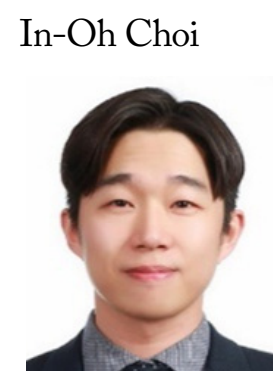

received his B.S. and M.S. degrees in electronic engineering from Pukyong National University, Busan, Korea, in 2012 and 2014, respectively, and his Ph.D. degree in electronic engineering from Pohang University of Science and Technology (POSTECH), Pohang, Korea, in 2019, respectively. From 2019 to 2021, he was a senior researcher with the Agency for Defense Development. In 2021, he joined the faculty of the Department of Electronics and Communications Engineering, Korea Maritime \& Ocean University, Busan, Korea, where he is currently a assistant professor. His current research areas of interest include micro-Doppler analysis, ballistic target discrimination, vital sign detection, automotive target recognition, and calibration of polarimetric synthetic aperture radar.

\section{Sang-Hong Park}

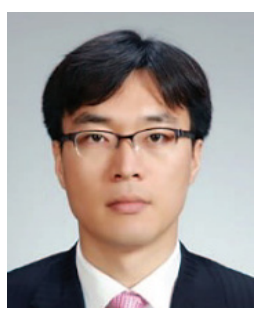

received his B.S., M.S., and Ph.D. degrees in electronic engineering from Pohang University of Science and Technology (POSTECH), Pohang, Korea, in 2004, 2007, and 2010, respectively. In 2010, he was a Brain Korea 21 Postdoctoral Fellow at the Electromagnetic Technology Laboratory, POSTECH. In 2010, he joined the faculty of the Department of Electronics Engineering, Pukyong National University, Busan, Korea, where he is currently a professor. His research interests are radar target imaging and recognition, radar signal processing, target motion compensation, and radar cross-section prediction. 